\title{
Hypokalemic Periodic Paralysis With Reversible Electrophysiological Abnormalities: A Case Report
}

\author{
Shoaib Bhattia , Tahniyat Huq ${ }^{\mathrm{a}}$, Syed Mohammad Mazhar Uddin ${ }^{\mathrm{b}}$, Aatera Haq ${ }^{\mathrm{b}}$, \\ Syed Faheem Ali Kazmic ${ }^{c}$, Uzair Yaqoob ${ }^{c}$,, Haider Shah ${ }^{\mathrm{c}}$
}

\begin{abstract}
Hypokalemic periodic paralysis (HPP) is a reasonably rare condition, presenting complains being those associated with acute systemic weakness and hypokalemia. Majority of the cases are characterized as familial or primary, whereas sporadic cases are associated with various other conditions such as barium poisoning, hyperthyroidism, renal disorders, endocrinopathies and gastrointestinal potassium losses. We report a unique case in a 5-year-old female child following gastrointestinal losses (vomiting and diarrhea). According to the patient's mother, for the past 2 days, she was lethargic, unable to walk and hold the neck. She underwent pyelolithotomy for recurrent kidney stones 5 years back. Her neurologic examination revealed diminished bulk overall, decreased tone, power and reflexes in lower limbs with down-going plantars. Labs (except potassium) and electrocardiography were normal. Nerve conduction studies (NCS) favored the diagnosis of Guillain-Barre syndrome. Intravenous immunoglobulin and potassium replacement was started. After a week, patient's condition had a remarkable improvement. We did a repeat NCS which came out to be normal. Potassium serum values were normal too. We turned back to the other differential diagnosis of HPP. Our case report highlights several important factors. Firstly, it states that the NCS can be deceptive in patients with flaccid paralysis, especially when we are not suspecting hypokalemia. It further explains the importance of suspecting a diagnosis of HPP in a patient presenting with features of Guillain-Barre syndrome following an NCS.
\end{abstract}

Keywords: Hypokalemia; GBS; Nerve conduction studies; Hypokalemic periodic paralysis

\section{Introduction}

Hypokalemic periodic paralysis (HPP), the most common

Manuscript submitted April 9, 2018, accepted May 3, 2018

${ }^{a}$ National Institute of Child Health, Karachi, Pakistan

${ }^{\mathrm{b} C i v i l ~ H o s p i t a l ~ K a r a c h i, ~ K a r a c h i, ~ P a k i s t a n ~}$

'Dow University of Health Sciences, Karachi, Pakistan

${ }^{\mathrm{d} C}$ Corresponding Author: Uzair Yaqoob, Dow University of Health Sciences,

Karachi, Pakistan. Email: ozair_91393@hotmail.com

doi: https://doi.org/10.14740/jmc3058w form of periodic paralysis, is reasonably rare with an approximated prevalence of 1 in 100,000 [1]. The presenting complains are usually associated with acute systemic weakness and hypokalemia. Majority of the cases are characterized as familial or primary HPP, whereas sporadic cases are associated with various other conditions such as barium poisoning, hyperthyroidism, renal disorders, endocrinopathies and gastrointestinal potassium losses. As far as management is concerned, immediate replacement of potassium is done and the exploring of the underlying etiology is initiated [2]. We report a unique case of hypokalemic paralysis with reversible electrophysiological abnormalities in a 5-year-old female child following gastrointestinal losses.

\section{Case Report}

A 5-year-old female, vaccinated, and developmentally normal, came to the emergency department with complains of diarrhea and vomiting since 10 days. According to the patient's mother, for the past 2 days, she was lethargic, unable to walk and also acquired inability to hold the neck. There was no associated fever or any other complains. Past medical and past surgical history was significant for recurrent kidney stones for which she had undergone pyelolithotomy in 2013. On examination, her vitals were blood pressure $80 / 54 \mathrm{~mm} \mathrm{Hg}$, pulse $100 / \mathrm{min}$, respiratory rate $30 / \mathrm{min}$, and temperature $36.7^{\circ} \mathrm{C}$. Furthermore, her weight was $11 \mathrm{~kg}$, height was $96 \mathrm{~cm}$ and FOC was $44 \mathrm{~cm}$. Her neurologic examination revealed an overall diminished bulk in both upper and lower extremities and a decreased tone in both her lower limbs, whereas the tone in her upper limbs was normal. Moreover, the power was $3 / 5$ and $5 / 5$ in her lower and upper limbs respectively and the reflexes were diminished in the lower limbs with down going plantars. At this point in time, based on the history and examination, we suspected Guillain-Barre syndrome (GBS) or HPP due to gastrointestinal losses, ordered her baseline laboratory tests as well as electrocardiography (ECG) and nerve conduction studies (NCS). Her baseline laboratory values came out to be hemoglobin $11.1 \mathrm{~g} /$ $\mathrm{dL}$, TLC $23.7 \times 10^{9} / \mathrm{L}$, platelet $575,000 / \mathrm{L}$, creatinine $0.3 \mathrm{mg} /$ $\mathrm{dL}$, sodium $139 \mathrm{mEq} / \mathrm{L}$, potassium $2.1 \mathrm{mEq} / \mathrm{L}$, and chloride $101 \mathrm{mEq} / \mathrm{L}$. Her ECG was normal and NCS was said to be abnormal with low compound muscle action potential (CMAP) amplitudes of median, ulnar and peroneal nerves. Furthermore, her left median, ulnar and sural sensory nerve action potential amplitude (SNAP) were normal along with normal 
F-wave latency from the posterior tibial nerve but the ulnar, median and peroneal F-waves were non-recordable. The findings were suggestive of pure motor neuropathy, and can be axonal in nature, favoring the diagnosis of GBS, possibly acute motor axonal neuropathy (AMAN) variant.

Given the calamity of the situation, we immediately shifted the patient to pediatric intensive care unit (ICU) where intravenous antibiotics were given and injection intravenous immunoglobulin (IVIG) $0.4 \mathrm{~g} / \mathrm{kg}$ intravenous (once a day for 2 days) was started slowly under strict blood pressure monitoring. Meanwhile, her potassium was also being replaced. After a week, patient's condition had a remarkable improvement as she was able to walk and hold her neck properly. Her base line laboratory values also improved with hemoglobin $11.5 \mathrm{~g} /$ $\mathrm{dL}$, TLC $10 \times 10^{9} / \mathrm{L}$, platelets $57,500 / \mathrm{L}$, creatinine $0.3 \mathrm{mg} / \mathrm{dL}$, sodium $137 \mathrm{mEq} / \mathrm{L}$, chloride $100 \mathrm{mEq} / \mathrm{L}$ and potassium 3.5 $\mathrm{mEq} / \mathrm{L}$. Keeping in view of the improvement, we did a repeat NCS and the findings showed right tibial and deep peroneal motor nerves having normal amplitude, velocities and distal latencies. Furthermore, F latencies were normal as well and right median and ulnar motor nerves had normal amplitudes, velocities, and distal latencies. Moreover, right sural, median and ulnar sensory nerves also had normal amplitudes, velocities and distal latencies, suggesting a normal study. Hence, this allowed us to revert back to our differential diagnosis of HPP.

\section{Discussion}

HPP is designated with reversible attacks of muscle weakness corresponding with low potassium levels. Deranged levels of potassium are observed between the attacks with muscle weakness oscillating parallel to the blood potassium levels. Furthermore, hypokalemia induces muscle depolarization, which is regarded as the main mechanism behind the muscle weakness [1]. These attacks usually affect the four limbs; however when incomplete, they can prevail in the lower limbs. Respiration, deglutition and ocular motility are usually affected only in severe attacks. Furthermore, studies have shown HPP does not affect the heart, but can trigger cardiac arrhythmias. Very rarely, patients can develop permanent weakness but this phenomenon is usually observed in young adults [1]. In our patient, only the lower limbs and neck muscles were involved, without any effect on respiration, deglutition and ocular motility. Moreover, our patient had a normal ECG as well as no sign of permanent weakness.

Several studies have reported that electrophysiological abnormalities observed in HPP include a diminished CMAP amplitude during a paralytic attack, raised CMAP amplitude 5 min after maximal muscle contraction, progressive reduction in amplitude 20 - 40 min after rest and very rarely, reduced SNAP can also be reported [3]. Numerous studies conducted in adult population have also shown severe reductions in the amplitudes of CMAP along with non-recordable F-wave latencies and normal sensory conduction studies. They have also stated a comprehensive improvement in the CMAP amplitudes along with normalization of F-wave latencies with the prompt administration of potassium, leading to improvement in motor power as early as the first $24 \mathrm{~h} \mathrm{[3].} \mathrm{However,} \mathrm{in}$ the pediatric population, to our knowledge, only a single case report before this was reported which showed a diminished amplitude in the CMAP with progressive improvement in the CMAP amplitudes after potassium administration [4]. Our patient's initial NCS also showed a diminished CMAP and non-recordable F-wave latencies (ulnar, medial and peroneal nerves) which improved following a serial NCS after potassium administration. Hence, our case report further adds value to the literature.

\section{Conclusion}

Overall, HPP can be considered as a rare entity, which should have a special place in the differential diagnosis of systemic weakness. The diagnostic approach to a patient with such a disease must include a robust exploration along with administration of potassium replacement therapy. Our case report highlights several important factors. Firstly, it states that the NCS can be deceptive in patients with flaccid paralysis, especially when we are not suspecting hypokalemia. It further explains the importance of suspecting a diagnosis of HPP in a patient presenting with features of AMAN variant of GBS following an NCS.

\section{References}

1. Fontaine B. Chapter 1 Periodic Paralysis. Advances in Genetics. 63: Academic Press; 2008. p. 3-23.

2. Ahlawat SK, Sachdev A. Hypokalaemic paralysis. Postgrad Med J. 1999;75(882):193-197.

3. Sharma CM, Nath K, Parekh J. Reversible electrophysiological abnormalities in hypokalemic paralysis: Case report of two cases. Ann Indian Acad Neurol. 2014;17(1):100-102.

4. Rajshekher G, Kumar S, Prabhakar S. Reversible electrophysiological abnormalities in hypokalemic periodic paralysis. Indian Pediatr. 2008;45(1):54-55. 